\title{
MÉTODOS DE ANÁLISE QUANTITATIVA APLICADOS A MODE- LOS DE CORROSÃO VASCULAR: REVISÃO DE LITERATURA
}

\author{
METHODS OF QUANTITATIVE ANALYSIS APPLIED TO \\ VASCULAR CORROSION CASTS: A LITERATURE REVIEW
}

Aderson Gegler ${ }^{1}$, Juliana Gazolla², Maria A.L. de Souza ${ }^{3}$

1'Doutor em Odontologia, Área de concentração Estomatologia, Hospital São Lucas da Pontifícia Universidade Católica do Rio Grande do Sul. ${ }^{2}$ Mestre em Periodontia, Universidade Luterana do Brasil. ${ }^{3}$ Doutora em Estomatologia, Professora e Coordenadora do Departamento de Ciências Morfológicas da Faculdade de Biociências, Pontifícia Universidade Católica do Rio Grande do Sul.

CoRRESPONDÊNCIA: Aderson Gegler. Rua Dario Pederneiras, n 41/703. 90630-090 - Porto Alegre / RS. email:adersongegler@hotmail.com

Gegler A, Gazolla J, Souza MAL. Métodos de análise quantitativa aplicados a modelos de corrosão vascular: revisão de literatura. Medicina (Ribeirão Preto) 2008; 41 (1): 77-85.

RESUMO: Os modelos de corrosão vascular propiciam um poderoso entendimento morfológico da microarquitetura dos vasos sangüíneos quando associados à microscopia eletrônica de varredura. Diversos sistemas de morfometria bidimensionais (2D) e tridimensionais (3D) têm sido propostos para a sua quantificação, bem como modelos matemáticos com o objetivo de entender os princípios ideais hemodinâmicos que governam estes sistemas circulatórios. Este artigo se propõe a realizar uma revisão de literatura desta evolução, desde as primeiras teses sobre os princípios ideais, fundamentais para o entendimento da hemodinâmica vascular, até o estado da arte dos sistemas de morfometria 3D.

Descritores: Molde por Corrosão. Microscopia Eletrônica de Varredura. Análise Quantitativa. Vasos Sangüíneos. Morfologia.

\section{1- INTRODUÇÃO}

O sistema circulatório é responsável pela condução e distribuição de oxigênio e dióxido de carbono para os tecidos, produtos do sistema endócrino, das trocas metabólicas com as células, transporte dos componentes inflamatórios e do reparo tecidual, além de ser coadjuvante na termorregulação (vasodilatação e vasoconstrição). Assim, o interesse pelo estudo desse sistema é bastante antigo. Em 1926, a partir da biologia teórica, foram desenvolvidos uma série de cálculos para encontrar a energia total da circulação sangüínea em uma determinada seção do sistema. ${ }^{1,2} \mathrm{Su}-$ geriu também a existência de uma lei teórica para a formação de ramificações arteriais com ângulos específicos.
Com o avanço científico e tecnológico, houve a possibilidade de aprimoramento e teste de tais teorias, inclusive permitindo a observação da circulação capilar. Em grande parte isso se deveu ao fato de os modelos de corrosão vascular começarem a ser analisados por meio de microscopia eletrônica de varredura. ${ }^{3}$ Além disso, com o desenvolvimento da microinformática, avanços dos sistemas de computação e das máquinas tiveram papel imprescindível para o aprimoramento de técnicas de análise quantitativa dos sistemas vasculares. Ainda assim, até os dias atuais existem algumas dificuldades tecnológicas para a execução de estudos quantitativos, fazendo com que grande parte das publicações na área seja de pesquisas com ênfase na análise qualitativa destes sistemas.

A importância das análises quantitativas fica 
evidente pela necessidade de conhecimento dos sistemas circulatórios. A partir deste conhecimento é possível comparar diferentes tecidos, órgãos, espécies, bem como respostas a tratamentos diferentes de determinada doença ou ainda a relação de desenvolvimento dessas doenças com o sistema circulatório. Esses dados juntamente com estudos de fatores de crescimento vasculares e tumorais podem ser uma informação extremamente útil para a evolução da pesquisa do câncer, já que existe uma correlação entre angiogênese e progressão tumoral. ${ }^{4}$ Desta forma, este estudo tem por objetivo revisar a literatura disponível sobre as diversas técnicas de quantificação dos sistemas circulatórios, ainda pouco utilizadas no Brasil, dando maior suporte a estudos posteriores utilizando a técnica de modelos de corrosão vascular associada à microscopia eletrônica de varredura (MEV).

\section{2- REVISÃO DA LITERATURA}

A lei de Poiseuille, ${ }^{5}$ de 1840 , relativa à mecânica dos fluidos, foi utilizada como base na formulação de teorias e equações matemáticas ${ }^{1,2}$ na tentativa de explicar as leis dos sistemas vasculares. Essa lei permite determinar, matematicamente, o fluxo laminar de um fluido viscoso através de um tubo cilíndrico de secção transversal circular. A energia total envolvida na circulação sangüínea em um segmento arterial foi equacionada e posteriormente foi desenvolvido um princípio hipotético sobre a ramificação arterial. ${ }^{1,2}$ Supondo que a energia total do sistema circulatório em questão seja mínima, a soma dos cubos dos raios das ramificações arteriais será igual ao cubo do raio da artéria da qual eles derivam $\left(r_{0}^{3}=r_{1}^{3}+r_{2}^{3}\right)$. Nesse contexto, também foi publicado que, quanto maior o diâmetro da ramificação arterial, menor o ângulo entre o ramo e a direção da artéria principal e que dois ramos de igual diâmetro terão ângulos iguais formados com o prolongamento da artéria mãe. ${ }^{1,2}$ Referente a esse conceito, é importante ressaltar que existe um razoável consenso de que o modo mais freqüente de ramificação arterial é o dicotômico, onde a artéria mãe se divide em somente duas artérias filhas. ${ }^{6}$

Em outra frente de estudos relacionada a sistemas circulatórios, surgiu a técnica de corrosão vascular, inicialmente utilizada em estudos macroanatômi$\cos ^{7,8,9}$ Porém, já em 1971 Murakami $^{3}$ descreveu pela primeira vez esta técnica, inicialmente com intuito de observação da microanatomia vascular em MEV. Este autor utilizou uma resina à base de metil metacrílico, a qual era injetada no sistema vascular do animal em estudo. Após a injeção e polimerização da resina, o órgão injetado era imerso em uma solução de hidróxido de sódio para a corrosão dos tecidos orgânicos, obtendo-se uma cópia fiel do sistema circulatório moldado. Posteriormente, a estrutura era coberta com camadas de carbono e ouro, em uma câmara de evaporação, para permitir a visualização no microscópio eletrônico de varredura. Com essa técnica os autores puderam obter e visualizar modelos vasculares de quase todo o animal sem a quebra de vasos, incluindo as circulações arterial, venosa e capilar. Além disso, a técnica permitiu um grande avanço no estudo anatômico microvascular, devido à grande profundidade de campo e alta resolução da MEV. A técnica de corrosão vascular foi sendo modificada ao longo dos anos. Novos materiais foram testados e os parâmetros das diversas fases do processo foram adaptados para diferentes animais, órgãos e tecidos. Uma visão completa da técnica, com as características das resinas, montagem dos espécimes, metalização, parâmetros físicos para a MEV e todas as fases para realização da técnica podem ser vistos em revisões específicas sobre o assunto. ${ }^{10 / 13}$

A partir do início dessa associação entre a técnica de corrosão vascular e a $\mathrm{MEV},{ }^{3}$ tornou-se viável o aprimoramento e o teste da validade de princípios fisiológicos sobre o desenvolvimento dos sistemas vasculares. Em $1976^{14}$ e $1978^{15}$ foi compilado que o ângulo formado entre o prolongamento da artéria principal com as ramificações (Figura 1) é tal que a superfície e o volume luminal dos vasos envolvidos em uma junção arterial são mínimos. Por conseguinte, o ângulo ideal dessa ramificação é determinado pelo raio dos vasos envolvidos. Outros dois princípios propõem que o ângulo da ramificação é tal que a força de bombeamento requerida para levar o fluxo ou a força de atrito entre o sangue e as superfícies endoteliais são mínimas. Desta forma, o ângulo ideal depende tanto do fluxo quanto do raio dos vasos envolvidos. A justificativa para atingir esses princípios ideais é dada pela liberdade de crescimento dos vasos durante sua formação. Entende-se por "ideais" valores de ângulos e diâmetros nos quais uma bifurcação possa ser eficiente, ou seja, apresentando uma das possíveis características que regem cada um dos diferentes "princípios ideais". Para a obtenção das fórmulas matemáticas desses princípios, ${ }^{15}$ foi utilizado o conceito de razão de área $\beta$, ou seja, a razão da soma das áreas da seção transversal dos vasos filhos pela área do vaso mãe, gerando a seguinte fórmula: 


$$
\beta=\frac{r_{1}^{2}+r_{2}^{2}}{r_{0}^{2}},
$$

onde $r_{0}$ é o diâmetro do vaso principal, $r_{1}$ da ramificação maior e $r_{2}$ da menor. Utilizou-se ainda, o conceito de índice de bifurcação $\alpha$ :

$$
\alpha=\frac{r_{2}}{r_{1}},
$$

sendo que o índice $\alpha$ pode variar de próximo de 0 (zero), muito assimétrico, a 1 (um), simétrico. Desta forma, quanto maior o índice $\alpha$, maior a simetria da bifurcação.

Considerando que o fluxo na artéria principal é igual a soma do fluxo nos dois $\operatorname{ramos}\left(f_{0}=f_{1}+f_{2}\right)$ e este sendo proporcional ao cubo do raio $\left(f \propto r^{3}\right)$, então $r_{0}^{3}=r_{1}^{3}+r_{2}^{3}$.
Por meio de deduções matemáticas e utilizando princípios trigonométricos, ${ }^{15,16}$ foram propostas equações para predizer os ângulos ideais das bifurcações governadas por cada um dos quatro princípios ideais, ao qual um sistema vascular pode estar submetido (maiores detalhes em Zamir $\left(1978^{15}\right.$ e $\left.1988^{16}\right)$ ). Em um princípio ideal baseado em minimizar o volume luminal e a força de bombeamento necessária temos:

$$
\begin{aligned}
& \cos \theta_{1}=\frac{\left(1+\alpha^{3}\right)^{4 / 3}+1-\alpha^{4}}{2\left(1+\alpha^{3}\right)^{2 / 3}} \mathrm{e} \\
& \cos \theta_{2}=\frac{\left(1+\alpha^{3}\right)^{4 / 3}-1+\alpha^{4}}{2 \alpha^{2}\left(1+\alpha^{3}\right)^{2 / 3}}
\end{aligned}
$$

enquanto que num princípio baseado em minimizar superfície luminal e a força de atrito temos:

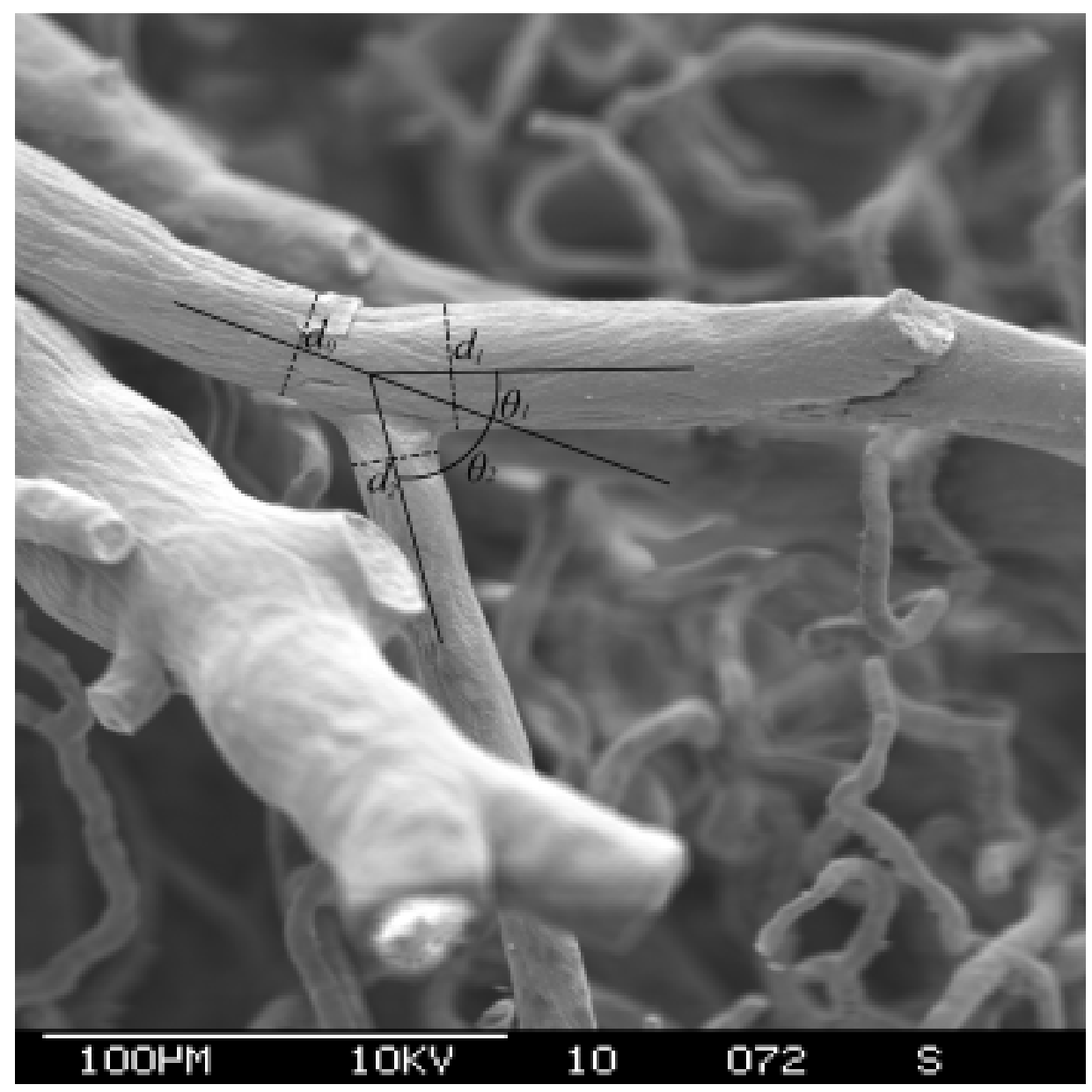

Figura 1: Bifurcação arterial de glândula submandibular de rato, $d_{0}$ representa o diâmetro do vaso mãe, $d_{1}$ do maior vaso filho e $d_{2}$ do menor vaso filho. $\theta_{1}$ e $\theta_{2}$ são os ângulos com os quais os vasos filhos, respectivamente, maior e menor, ramificam-se do vaso mãe. $350 \mathrm{X}$, distancia de trabalho $10 \mathrm{~mm}$. 


$$
\begin{aligned}
& \cos \theta_{1}=\frac{\left(1+\alpha^{3}\right)^{2 / 3}+1-\alpha^{2}}{2\left(1+\alpha^{3}\right)^{1 / 3}} \mathrm{e} \\
& \cos \theta_{2}=\frac{\left(1+\alpha^{3}\right)^{2 / 3}-1+\alpha^{2}}{2 \alpha\left(1+\alpha^{3}\right)^{1 / 3}}
\end{aligned}
$$

Uma bifurcação sob as características de um dos quatro princípios ideais gera um custo de energia mínimo para o sistema, contudo, qualquer desvio geométrico gera um custo mais alto, devido a perda de eficiência hemodinâmica. ${ }^{17}$ É notável que, mesmo com uma considerável mudança nos ângulos das ramificações, o custo para o sistema não é linearmente proporcional e está dentro de uma faixa de até $2 \% .{ }^{17}$ Os princípios ideais já foram utilizados, também, para predizer ângulos de ramificações em árvores, ${ }^{18}$ explicar a geometria do vérmis cerebelar humano ${ }^{19}$ e a geometria de drenagem dos leitos fluviais ${ }^{20}$.

Outro conceito discutido na literatura refere-se ao tipo de sistema vascular, o qual pode ser de distribuição ou de entrega. ${ }^{21}$ As características de um sistema de distribuição mostram um vaso que não entra em uma região específica, mas transporta o sangue para várias zonas diferentes. Já um sistema de entrega, adentra uma pequena região exatamente para prover o suprimento sangüíneo dela. Assim, um sistema de distribuição terá um resultado de razão de ramificação mais baixo e mostrará menor número de bifurcações, enquanto que o sistema de entrega mostrará uma razão de ramificação mais alta e será possível observar vasos se dividindo mais profusamente, porém terminando mais rapidamente. Geralmente, vasos de distribuição têm um calibre maior do que os de entrega, porém não para todos os casos. Esses conceitos podem ser relevantes quando se necessita determinar o grau de importância funcional de um sistema.

A análise quantitativa também seguiu outro caminho, com um foco mais anatômico e não tão fisiológico, realizando diversas formas de mensurações sobre as fotomicrografias resultantes da MEV. Um método proposto foi o de contagem de pontos, o qual emprega os princípios da estereologia, sendo utilizado na avaliação de redes vasculares planas ou seccionadas. ${ }^{22}$ Uma grade-teste é sobreposta à micrografia ou diretamente à tela do microscópio eletrônico de varredura para, então, serem feitos os cálculos, utilizando-se as fórmulas estereológicas. Já a estereofotogrametria necessita de um estereoscópio equipado com um sistema de medidas que aplique o princípio da paralaxe para a aquisição de imagens na $\mathrm{MEV}^{23}$

A proposta da planimetria, associada a um computador, é um método simples destinado à análise bidimensional. Consiste na mensuração do comprimento dos vasos traçando-se uma linha no centro dos mesmos. O tamanho final desses vasos é calculado por um programa de computador. ${ }^{24}$ Ainda, outra forma bidimensional de morfometria vascular é realizada por meio da análise de imagens que estão ou não em foco. As imagens dos vasos que estão em foco são desenhadas em branco e os vasos que se apresentam fora de foco, em preto. ${ }^{25}$ Analogamente, isso foi realizado também utilizando um programa de computador, ${ }^{26}$ onde visualmente foi escolhido um ponto de corte para os níveis de cinza da imagem resultante da MEV (threshold). Os níveis de cinza superiores a este ponto seriam ajustados para brancos (referem-se à área vascular) e inferiores, para pretos (referem-se à área avascular), criando assim uma imagem binária. A partir dessas duas metodologias, que fornecem a proporção de área vascular e avascular, é calculada a razão entre a área branca e a área total, obtendo-se um índice de densidade vascular. ${ }^{25,26}$

Em alguns estudos que empregam o princípio de análise de tons de cinza, ${ }^{27,28}$ a seleção das áreas foi feita com a ferramenta threshold de um programa de edição de imagens. Da mesma forma, o princípio de diferença nos tons de cinza entre imagens em foco e fora dele foi utilizado. Com o intuito de minimizar o problema da representatividade dos níveis de cinza quanto à profundidade das camadas vasculares, foi sugerido o uso de outra ferramenta digital de tratamento de imagem, o laço magnético. ${ }^{29}$ Neste caso, um operador deve selecionar o contorno dos vasos a serem analisados, antes de transformar a imagem em binária, para se realizar o cálculo da densidade vascular. Neste estudo foram testados diversos algoritmos para ajuste das imagens, com o objetivo de encontrar uma forma que mais se aproxime do padrão-ouro também calculado.

Todas essas técnicas de morfometria referidas têm limitações, seja pela forma de mensuração e tipo de resultado, seja pelo grau de fidelidade que guardam com objeto real. Dessa forma, sistemas tridimensionais (3D) de morfometria foram desenvolvidos, ${ }^{30,31,32}$ possibilitando a obtenção de medidas lineares e angulares acuradas. A diferença destes para os sistemas bidimensionais é a correção das medidas obtidas por algoritmos específicos utilizando-se do princípio da 
paralaxe (genericamente $[1 / \cos (\hat{a}$ ngulo de inclinação)])..$^{33}$ Em uma análise bidimensional, necessita-se de duas coordenadas para cada ponto $\left(X^{\prime} ; Y^{\prime}\right)$, porém em análises tridimensionais, necessita-se de três $\left(\mathrm{X}^{\prime} ; \mathrm{Y}^{\prime} ; \mathrm{Z}^{\prime}\right)$, sendo a terceira coordenada a profundidade do conjunto. Para que isso seja possível, é necessária uma modificação nos padrões de aquisição das imagens no MEV. São adquiridas duas imagens do mesmo sítio, com um intervalo angular de $6^{\circ} 30,32$ ou $8^{\circ} 31$ entre as mesmas, com a mesma distância de trabalho (10mm). Essas imagens são rotadas em $90^{\circ}$ no sentido anti-horário, como correção ao plano de orientação da visão humana. As imagens são coloridas, com intuito de uma visualização qualitativa, uma de verde e outra de vermelho, através de modificação dos canais $R G B$ (Red, Green, Blue). Estas imagens são, então, sobrepostas por um programa de computador, gerando uma imagem estereopareada passível de visualização com óculos verde/vermelho. ${ }^{32}$ Outra forma de observação de imagens anaglíficas é através de óculos estéreo com as imagens não sobrepostas, permitindo que cada olho visualize apenas uma imagem, ou ainda a visualização do par em uma tela, alternando-as rapidamente. ${ }^{30}$

Para a obtenção de medidas lineares e angulares, um dos sistemas ${ }^{30}$ propôs em seus algoritmos o uso dos princípios do Teorema de Pitágoras. Neste caso, uma distância $d$ entre dois pontos $P 1$ e $P 2$ pode ser encontrada pelo uso das coordenadas ( $X^{\prime}, Y^{\prime}$, e Z'), na equação:

$d=\sqrt{\left[\left(P 1_{X}+P 2_{X}\right)^{2}+\left(P 1_{Y}+P 2_{Y}\right)^{2}+\left(P 1_{Z}+P 2_{Z}\right)^{2}\right]}$ desde que se conheça o ângulo de inclinação entre as duas imagens $\left(6^{\circ}\right)$. O ângulo $\psi$ formado entre as ramificações pode ser calculado pela regra dos cossenos, com a equação $\psi=\arccos \left[\left(b^{2}+c^{2}-a^{2}\right) /(2 . b . c)\right]$, onde $b$ e $c$ são segmentos mensurados dos vasos em questão e $a$, a distância entre eles na extremidade oposta à ramificação. Este método permite um erro dimensional máximo de 2,5\%.

Com o mesmo objetivo, no programa 3DMorphometry® (Minnich \& Muska, OEG, Salzburg, Áustria), ${ }^{32}$ um operador marca pontos correspondentes em cada uma das duas imagens separadamente. Considere um ponto $P$, com suas coordenadas planas $(\mathrm{X}, \mathrm{Y})$ na primeira imagem (inclinação $\left.0^{\circ}\right) P_{0}(\mathrm{u}, \mathrm{v}) \mathrm{e}$ suas coordenadas correspondentes na segunda imagem (inclinação $\left.6^{\circ}\right) P_{6}(\mathrm{~s}, \mathrm{t})$. No caso de uma projeção assim chamada central, ou seja, em magnificações menores ou iguais a 500X, quando a relação entre dis- tância de trabalho e magnificação da imagem é tal que uma maior área do espécime é concebida no campo de visão do observador (câmera CCD - charge coupled device - do MEV) e o ângulo de visão é maior, obtém-se as coordenadas espaciais ( $X^{\prime}, Y^{\prime}$ e Z') dos pontos pelos seguintes algoritmos:

$X=\frac{10}{N} \cdot\left(4 \operatorname{sv}(\operatorname{sen}(\gamma) \operatorname{sen}(\rho / 2))^{2}+s \operatorname{sen}(2 \gamma) \operatorname{sen}(\rho)\right)$,

$Y=\frac{10}{N} \cdot((t+v) \operatorname{sen}(\gamma) \operatorname{sen}(\rho))$,

$Z=\frac{10}{N} \cdot\left(4 t v(\operatorname{sen}(\gamma) \operatorname{sen}(\rho / 2))^{2}+(t-v) \cos (\gamma) \operatorname{sen}(\rho)\right)$ sendo que:

$\mathrm{N}=\operatorname{sen}(2 \gamma)(1+\cos (\rho))+t v \operatorname{sen}(2 \gamma)(1-\cos (\rho))+$ $(t-v) \cos (2 \gamma) \operatorname{sen}(\rho)$, onde $\gamma$ é o ângulo de inclinação entre as imagens e $\rho$ o ângulo de abertura do campo. Já para projeções chamadas paralelas, conseqüência de magnificações maiores de 500X, onde se observa uma menor área do espécime no mesmo campo de visão, os algoritmos são:

$X=\frac{\mu s}{2}, Y=\mu \frac{t+v}{4 \cos (\gamma)}, Z=\mu \frac{t-v}{4 \operatorname{sen}(\gamma)}$

onde $\mu$ é o comprimento lateral do campo de visão, em $\mu \mathrm{m}$ (tabela de valores em Minnich, 1999). ${ }^{32}$ Uma vez obtidas as coordenadas dos pontos das estruturas em questão, princípios básicos de trigonometria permitem calcular ângulos e distâncias entre eles. Nenhum outro sistema de morfometria 3D relata a correção para esse erro de perspectiva, ressaltandose o fato de que ele trabalha com um erro dimensional consideravelmente baixo, de no máximo $1 \%{ }^{32,34}$

Até o momento, mais dois sistemas de morfometria tridimensional para aplicação em modelos de corrosão vascular foram introduzidos. Mexx ${ }^{\circledR}$ (Alicona, Alemanha) ${ }^{34}$ e outro publicado por Manelli (2007), ${ }^{35}$ porém não há informações detalhadas disponíveis sobre sua acurácia e algoritmos utilizados. Existem ainda outros métodos de quantificação e morfometria tridimensional para uso diverso dos modelos de corrosão vascular associada à MEV. A exemplo disso citase a microtomografia, ${ }^{36,37}$ em que é injetada uma substância de contraste no sistema vascular, não sendo necessária a corrosão dos tecidos orgânicos. Os algoritmos utilizados nestes programas são bastante poderosos podendo, inclusive, calcular o volume de estruturas. Porém a resolução permanece menor do que a alcançada com a MEV, $20 \mu \mathrm{m}$ (referente às di- 
mensões laterais de 1 voxel) e 3,5nm (Philips ESEM XL30®, FEI Company, Eindhoven, Holanda), respectivamente. Há ainda o método semi-automatizado de delineamento, ${ }^{38}$ que necessita um marcador para o plasma, como substância de contraste, antes da aquisição das imagens. Contudo, estes métodos não são aplicados a modelos de corrosão vascular associados à MEV. Da mesma forma, outros métodos de avaliação da macroarquitetura vascular para modelos de corrosão vascular relatados na literatura não serão abordados neste estudo, pois fogem do escopo desta revisão.

\section{3- DISCUSSÃo}

Desde as significativas contribuições para o estudo hemodinâmico e anatomo-funcional dos sistemas vasculares introduzidos por Murray em $1926^{1,2}$ até os grandes avanços teóricos da década de 70 e $80,,^{6,14 / 17}$ não havia a possibilidade de se testar ou utilizar essas teses, devido a uma lacuna entre conhecimento e tecnologia disponível. As possibilidades eram o uso de análises bidimensionais como a contagem de pontos, ${ }^{22}$ estereofotogrametria, ${ }^{23}$ planimetria,${ }^{24}$ mensuração de razão de área por níveis de cinza, ${ }^{25 / 29}$. Estes métodos porém, não propiciavam respaldo matemático para testar ou aplicar tais teorias em sistemas circulatórios. Essa possibilidade surgiu a partir do vínculo entre a técnica de modelos de corrosão vascular e a $\mathrm{MEV},{ }^{3}$ juntamente com o avanço tecnológico das últimas décadas (sistemas de lentes de alta precisão, aquisição digital de imagens, computadores com maior capacidade de processamento, desenvolvimento de programas de computador, ${ }^{30,31,32}$ armazenamento de dados). Então sim, foi possível o teste ${ }^{37}$ e a possibilidade de aplicação destas teorias e princípios hemodinâmicos. ${ }^{37,39 / 42}$

Com a formulação e desenvolvimento dos princípios hemodinâmicos, houve grandes contribuições para o campo de estudo dos sistemas circulatórios. Passou-se a ter um melhor entendimento fisiológico sobre a dinâmica do suprimento sangüíneo, seu sistema de irrigação, desenvolvimento e comportamento dentro de órgãos ou tecidos específicos. ${ }^{6,16,17,36,37,40 / 43}$ Estudos quantitativos simples, sem a análise ou comparação das razões de área ou de assimetria, ou entre os princípios ideais, fornecem uma contribuição mais anatômica e estática desses sistemas, ${ }^{26,39,44,45}$ o que algumas vezes pode ser alcançado por sistemas bidimensionais.
Um aspecto importante a considerar é que, como se tratam de métodos de avaliação quantitativa, os modelos devem refletir o melhor possível o estado do sistema vascular in vivo. Assim, o material de moldagem deve ter algumas propriedades ideais, como viscosidade semelhante à do sangue, capacidade de cópia de impressões das células endoteliais e uma contração de polimerização não significativa, para que seja possível a realização de mensurações confiáveis. ${ }^{10,11}$ Nenhum material preenche plenamente todos esses critérios. Entretanto, a resina utilizada mais freqüentemente (Mercox, Ladd Research, Burlington, EUA) demonstra características aceitáveis para todos esses quesitos, permitindo que a contração de polimerização possa ser desconsiderada, por não interferir significativamente nas dimensões finais. ${ }^{10,11}$ Nesse sentido, ressalta-se, também, que as camadas de carbono e ouro utilizadas na metalização, para tornar o modelo condutível e visível ao MEV, não influenciam nas medidas realizadas, já que são da ordem de 15 a $400 \mathrm{~nm}^{.11}$

De acordo com os recursos de pesquisa disponíveis hoje, seria uma grande contribuição ao estudo hemodinâmico das espécies, órgãos ou tecidos e principalmente da angiogênese, a união dos conhecimentos disponíveis pela técnica de corrosão vascular associada à $\mathrm{MEV}$, sistemas morfométricos $3 \mathrm{D}$ digitais $\mathrm{e}$ princípios fisiológicos ideais, ao estudo dos fatores de crescimento. Isso devido ao fato de que, com o auxílio de teorias matemáticas sobre esses princípios ideais dos sistemas circulatórios, que começaram a surgir já em $1926^{1,2}$, também são possíveis pesquisas que contribuam para o entendimento da anatomia funcional e desenvolvimento de sistemas circulatórios. ${ }^{36}$ Assim como do custo de energia necessário para o organismo e a diferenciação entre sistema de distribuição ou entrega.

Alguns estudos ${ }^{37,40,42}$ mapearam em gráficos o resultado das mensurações das ramificações arteriais, para avaliar se as mesmas se inserem ou não em áreas consideradas ideais pelos princípios fisiológicos. Tal procedimento permite estabelecer quantitativamente o grau de envolvimento do sistema pesquisado em relação aos princípios ideais, conforme sugerido anteriormente. ${ }^{15}$ Porém, existem limitações no modelo matemático que devem ser consideradas. A natureza pulsátil do fluxo sangüíneo, a elasticidade e a não uniformidade da espessura da parede dos vasos e a sua curvatura longitudinal, são fatores não considerados. Entretanto, a ausência de modelos matemáti- 
cos mais sofisticados justifica o uso dos disponíveis, ${ }^{14}$ visto que ainda hoje estes são utilizados ${ }^{37,40,41,42,44}$ por falta de novas alternativas.

Sobre a eficiência dos métodos bidimensional e tridimensional, foi realizada uma análise comparativa na mensuração do comprimento vascular. ${ }^{46}$ Foi verificado que os comprimentos medidos na avaliação bidimensional sempre eram menores que os medidos na tridimensional, diferindo, em média, $12,89 \%$. Tal achado justifica-se pelo fato de que, para uma análise bidimensional, necessita-se de duas coordenadas para cada ponto $\left(X^{\prime} ; Y^{\prime}\right)$ e, para análises tridimensionais, de três $\left(X^{\prime} ; Y^{\prime} ; Z^{\prime}\right)$, sendo a terceira coordenada que determina a profundidade do conjunto. Assim, análises morfométricas confiáveis somente são alcançadas com sistemas tridimensionais, que dependem da coordenada Z', ou seja, a orientação espacial do conjunto. Isso não impede que, dependendo do tipo de dado que se busque, como por exemplo, a densidade vascular de uma região, seja possível a utilização de sistemas bidimensionais.

Levando-se em conta a evolução, tanto dos programas, quanto dos computadores, alguns desses métodos relatados na literatura são, hoje, obsoletos. Porém, a dificuldade na obtenção de recursos para análises tridimensionais em alguns centros de pesquisa justifica o desenvolvimento de ferramentas alternativas que possam suprir as necessidades das pesquisas nesta área. Esse fator fez com que surgissem métodos simples e confiáveis para análises quantitativas da vascularização em diferentes tecidos ou regiões anatômicas, quando da utilização da técnica de corrosão vascular. ${ }^{29}$

Há que se considerar, em relação à comparação entre MEV e microtomografia, que existem hoje microtomógrafos que alcançam uma resolução (dimensão de voxel) de até 150nm (SkyScan2011®, Skyscan, Kontich, Bélgica, 2007). ${ }^{47}$ Isto deixa precedentes para um grande campo de pesquisas, porém é importante considerar o custo desse tipo de equipamento. Outros microtomógrafos que alcançam uma resolução espacial considerada alta, dimensões do voxel de em torno de $1 \mu \mathrm{m}$ a $2 \mu \mathrm{m}($ SkyScan1172®, Skyscan, Kontich,
Bélgica, 2007), ${ }^{48}$ continuam com resultados inferiores ao MEV, onde ela é de até $2 \mathrm{~nm}\left(\mathrm{EVO}{ }^{\circledR} W D S\right.$, Carl Zeiss SMT AG, Oberkochen, Alemanha $)^{49}$ ou 3,5nm (Philips ESEM XL30®, FEI Company, Eindhoven, Holanda $)^{50}$. Sabe-se que, quando se utiliza uma magnificação de $250 \mathrm{X}$ a 500X, padrão nas aquisições de imagens de capilares para morfometria, essa resolução não é tão alta. Ainda assim, a MEV proporciona resultados bastante superiores para os casos em que o diâmetro total do capilar pode chegar em níveis próximos de $4 \mu \mathrm{m}$ a $11 \mu \mathrm{m} .{ }^{42}$ Isto certamente traria uma visualização prejudicada, ${ }^{40}$ se a resolução máxima do equipamento fosse de $3 \mu \mathrm{m}$, quando se tem por objetivo priorizar a morfometria.

Ainda, tecnicamente, é necessário considerar a relação entre magnificação e menor profundidade de campo, para o uso dos sistemas 3D. Como o objetivo são análises tridimensionais, isso implica em avaliação também da profundidade do conjunto. Será de fundamental importância para que se possa ter uma boa visualização desse conjunto, uma profundidade de campo razoável. Isso é alcançado com a combinação de algumas variáveis físicas no MEV, como a distância de trabalho, abertura e voltagem. Assim, o ajuste desses parâmetros permite que diversas camadas de vasos sejam visualizadas em uma mesma imagem. ${ }^{29}$

Assim, podemos considerar que uma grande quantidade de conhecimento, bem como sistemas de morfometria poderosos, estão disponíveis para aplicação no campo da hemodinâmica dos sistemas circulatórios, seu desenvolvimento, comparações entre diferentes tecidos, órgãos ou espécies e ainda entre tecidos sadios e doentes. A partir disto, se poderá buscar um melhor entendimento relativo ao desenvolvimento da angioarquitetura e suprimento nutricional de tumores e outras doenças.

\section{AGRADECIMENTOS}

O autor (A.G.) foi financiado por bolsas da CAPES no Programa de Doutorado em Odontologia PUCRS (42005019022D9) e Estágio de Doutorado no Exterior (BEX1335-06-5). 
Gegler A, Gazolla J, Souza MAL. Methods of quantitative analysis applied to vascular corrosion casts: a literature review. Medicina (Ribeirão Preto) 2008; 41 (1): 77-85.

ABSTRACT: Vascular corrosion casts combined with scanning electron microscopy provide a powerful morphologic understanding of the vascular microarchitecture. Several bidimensional (2D) and threedimensional (3D) systems, as well as mathematical models have been proposed aiming at the quantification of vascular models, in order to study the hemodynamic optimality principles ruling those circulatory systems. This paper aims to accomplish a literature review of this evolution from the foremost thesis about optimality principles, vital to the knowledge of vascular hemodynamics, up to the state-of-the-art 3D morphometry systems.

Keywords: Corrosion casting. Microscopy, Electron, Scanning. Quantitative Analysis. Blood Vessels. Morphology.

\section{REFERÊNCIAS BIBLIOGRÁFICAS}

1 - Murray CD. The Physiological Principle of Minimum Work: II. Oxygen Exchange in Capillaries. Proc Natl Acad Sci U S A. 1926 May;12(5):299-304.

2 - Murray CD. The Physiological Principle of Minimum Work: I. The Vascular System and the Cost of Blood Volume. Proc Natl Acad Sci U S A. 1926 Mar;12(3):207-14.

3 - Murakami T. Application of the scanning electron microscope to the study of the fine distribution of the blood vessels. Arch Histol Jpn. 1971 Feb;32(5):445-54.

4 - Folkman J. Tumor angiogenesis: therapeutic implications. N Engl J Med. 1971 Nov 18;285(21):1182-6.

5 - Poiseuille L. Poiseuille's law. 1840 [cited 12/09/2007]; Available from: http://en.wikipedia.org/wiki/Poiseuille's_law

6 - Zamir M, Phipps S. Network analysis of an arterial tree. J Biomech. 1988;21(1):25-34.

7 - Taniguchi $\mathrm{Y}$, Ohta $\mathrm{Y}$, Tajiri S. [New improved method for injection of acrylic resin.]. Okajimas Folia Anat Jpn. 1952 Oct;24(4):259-67.

8 - Taniguchi Y, Ohta Y, Tajiri S, Okano H, Hanai H. Supplement to new improved method for injection of acrylic resin. Okajimas Folia Anat Jpn. 1955 Dec;27(6):401-6.

9 - Batson OV. Corrosion specimens prepared with a new material. Anatomical Records. 1955;121:425.

10 - Weiger T, Lametschwandtner A, Stockmayer P. Technical parameters of plastics (Mercox CL-2B and various methylmethacrylates) used in scanning electron microscopy of vascular corrosion casts. Scan Electron Microsc. 1986;1(Pt 1):243-52.

11 - Lametschwandtner A, Lametschwandtner U, Weiger T. Scanning electron microscopy of vascular corrosion caststechnique and applications: updated review. Scanning Microsc. 1990 Dec;4(4):889-940; discussion 1.

12 - Verli FD, Kraether Neto L, Cherubini K, Souza MALd. Emprego da técnica de modelo de corrosão vascular em pesquisa odontológica. RFO UPF. 2006;11(1):7-12.
13 - Verli FD, Rossi-Schneider TR, Schneider FL, Yurgel LS, de Souza MA. Vascular corrosion casting technique steps. Scanning. 2007 May-Jun;29(3):128-32.

14 - Zamir M. The role of shear forces in arterial branching. J Gen Physiol. 1976 Feb;67(2):213-22.

15 - Zamir M. Nonsymmetrical bifurcations in arterial branching. $J$ Gen Physiol. 1978 Dec;72(6):837-45.

16 - Zamir M. The branching structure of arterial trees. Comments Theor Biol. 1988;1:15-37.

17 - Zamir M, Bigelow DC. Cost of departure from optimality in arterial branching. J Theor Biol 1984 Aug 7;109(3):401-9.

18 - Murray CD. A relationship between circumference and weight in trees and its bearing on branching angles. J Gen Physiol. 1927 May 20, 1927;10(5):725-9.

19 - Turner RS. The geometry of the human cerebellar vermis. Anat Rec 1968 Apr;160(4):691-6.

20 - Leopold LB. Trees and streams: the efficiency of branching patterns. J Theor Biol 1971 May;31(2):339-54.

21 - Zamir M. Distributing and delivering vessels of the human heart. J Gen Physiol 1988 May;91(5):725-35.

22 - Weibel ER. Morphometry of the human lung: the state of the art after two decades. Bull Eur Physiopathol Respir. 1979 Sep-Oct;15(5):999-1013.

23 - Mönnig B, El-Gammal S, Witsch P, Stanka P. Computer-aided three-dimensional reconstruction of terminal blood spaces in the proximal tibia metaphysic of the growing rat. VIII Intern Symp Morphol Sciences; 1988; Rome; 1988. p. 274.

24 - Kratky RG, Roach MR. Scanning electron microscopy of early atherosclerosis in rabbits using aortic casts. Scanning Microsc. 1988 Mar;2(1):465-70.

25 - Schraufnagel DE. Microvascular corrosion casting of the lung. A state-of-the-art review. Scanning Microsc. 1987 Dec;1(4):1733-47.

26 - Nelson AC. Study of rat lung alveoli using corrosion casting and freeze fracture methods coupled with digital image analysis. Scanning Microsc. 1987 Jun;1(2):817-22. 
27 - Borba MG. Angioarquitetura pulpar de incisivos de rats pela técnica de corrosão vascular. [Tese de Doutorado], Porto Alegre: Faculdade de Odontologia - PUCRS; 2004.

28 - Kraether Neto L. Angioarquitetura da superfície dorsal da língua de rats pelo método de corrosão vascular. [Tese de Doutorado], Porto Alegre: Faculdade de Odontologia - PUCRS; 2005.

29 - Gegler A, Lametschwandtner A, Cherubini K, Borba M, Souza $M$. Estimation of vascular areas in scanning electron micrographs of microvascular corrosion casts by 2Dmorphometry: a comparison of the magnetic lasso tool (Adobe Photoshop ${ }^{8} 7.0$ ) and the threshold tool (UTHSCSA ImageTool® 3.0). Em publicação 2008.

30 - Malkusch W, Konerding MA, Klapthor B, Bruch J. A simple and accurate method for 3-D measurements in microcorrosion casts illustrated with tumour vascularization. Anal Cell Pathol. 1995 Jul;9(1):69-81.

31 - Komatsu F, Tsubusaki K, Miyoshi M. Measurement of geometrical dimensions using scanning electron microscopy. J Electron Microsc (Tokyo). 1999 January 1, 1999;48(4):40715.

32 - Minnich B, Leeb H, Bernroider EW, Lametschwandtner A Three-dimensional morphometry in scanning electron microscopy: a technique for accurate dimensional and angular measurements of microstructures using stereopaired digitized images and digital image analysis. $\mathrm{J}$ Microsc. 1999 Jul;195(Pt 1):23-33.

33 - Kratky RG, Roach MR. Endothelial cell morphometry near branch junctions of rabbit aortae. Can J Physiol Pharmacol. 1987 Sep;65(9):1864-71.

34 - Minnich B, Krautgartner W, Lametschwandtner A. Quantitative 3D-Analyses in SEM: A Review. Microscopy and Microanalysis. 2003;9(Supplement S03):118-9.

35 - Manelli A, Sangiorgi S, Binaghi E, Raspanti M. 3D analysis of SEM images of corrosion casting using adaptive stereo matching. Microsc Res Tech. 2007 Apr;70(4):350-4.

36 - GössI M, Malyar NM, Rosol M, Beighley PE, Ritman EL. Impact of coronary vasa vasorum functional structure on coronary vessel wall perfusion distribution. Am J Physiol Heart Circ Physiol. 2003 Nov;285(5):H2019-26.

37 - Gössl M, Rosol M, Malyar NM, Fitzpatrick LA, Beighley PE, Zamir $\mathrm{M}$, et al. Functional anatomy and hemodynamic characteristics of vasa vasorum in the walls of porcine coronary arteries. Anat Rec A Discov Mol Cell Evol Biol. 2003 Jun;272(2):526-37.

38 - Barber PR, Vojnovic B, Ameer-Beg SM, Hodgkiss RJ, Tozer GM, Wilson J. Semi-automated software for the threedimensional delineation of complex vascular networks. J Microsc. 2003 Jul;211(Pt 1):54-62.

39 - Fait E, Malkusch W, Gnoth S-H, Dimitropoulou C, Gaumann A, Kirkpatrick CJ, et al. Microvascular Patterns of the Human Large Intestine: Morphometric Studies of Vascular Parameters in Corrosion Casts Scanning Microscopy. 1998;12(4):641-51.
40 - Lametschwandtner A, Minnich B, Kachlik D, Setina M, Stingl $\mathrm{J}$. Three-dimensional arrangement of the vasa vasorum in explanted segments of the aged human great saphenous vein: scanning electron microscopy and three-dimensional morphometry of vascular corrosion casts. Anat Rec A Discov Mol Cell Evol Biol. 2004 Dec;281(2):1372-82.

41 - Stottinger B, Klein M, Minnich B, Lametschwandtner A. Design of cerebellar and nontegmental rhombencephalic microvascular bed in the sterlet. Acipenser ruthenus: a scanning electron microscope and 3D morphometry study of vascular corrosion casts. Microsc Microanal. 2006 Oct;12(5):376-89.

42 - Kachlik D, Baca V, Stingl J, Sosna B, Lametschwandtner A, Minnich B, et al. Architectonic arrangement of the vasa vasorum of the human great saphenous vein. J Vasc Res. 2007;44(2):157-66.

43 - Aharinejad S, Schreiner W, Neumann F. Morphometry of human coronary arterial trees. Anat Rec. 1998 May;251(1):50-9.

44 - Cecon S, Minnich B, Lametschwandtner A. Vascularization of the brains of the Atlantic and Pacific hagfishes, Myxine glutinosa and Eptatretus stouti: a scanning electron microscope study of vascular corrosion casts. J Morphol. 2002 Jul;253(1):51-63.

45 - Minnich B, Bartel H, Lametschwandtner A. How a highly complex three-dimensional network of blood vessels regresses: the gill blood vascular system of tadpoles of Xenopus during metamorphosis. A SEM study on microvascular corrosion casts. Microvasc Res. 2002 Nov;64(3):425-37.

46 - Minnich B, Lametschwandtner A. Lengths measurements in microvascular corrosion castings: two-dimensional versus three-dimensional morphometry. Scanning. 2000 MayJun;22(3):173-7.

47 - SkyScan. SkyScan2011. 2007 [cited 2007 Set 28]. Available from: http://www.skyscan.be/products/2011.htm

48 - SkyScan. SkyScan1172. 2007 [cited 2007 Set 29]. Available from: http://www.skyscan.be/products/1172.htm

49 - EVO-WDS. Carl Zeiss, SMT AG. 2007 [cited 2007 Set 29] Available from: http://www.smt.zeiss.com

50 - ESEM-XL30. Philips, FEI Company 2007 [cited 2007 Set 29]. Available from: http://prism.mit.edu/facilities/XL30/ xl30home.htm

Recebido para publicação em 16/10/2007.

Aprovado para publicação em 12/11/2007. 